\title{
Diffuse axonal injury: pathological and clinical aspects
}

\begin{abstract}
Diffuse axonal and vascular injuries are the initial pathological findings in victims of diffuse brain trauma. Macroscopic findings include deep contusions and multiple petechial hemorrhages primarily involving cerebral white matter, basal ganglia and upper brainstem. Histologically $\beta$-amyloid precursor protein staining still remains the gold standard for microscopic detection of diffuse axonal damage. A number of other histological and immunohistochemical techniques have emerged over time aiding the diagnosis of diffuse axonal injury. There has been little success however in the microscopic differentiation of traumatic from non traumatic causes of diffuse axonal injury. This review explains various pathological and clinical aspects of traumatic axonal and vascular injuries including current scenarios in microscopic detection of diffuse axonal injury as well as their limitations.
\end{abstract}

Volume I Issue 4 - 2015

\author{
Pawan Mittal \\ Department of Forensic Medicine, BPS Government Medical \\ College for Women, India
}

Correspondence: Pawan Mittal,Assistant Professor, Department of Forensic Medicine, BPS Government Medical College for Women, Khanpur Kalan, Sonepat, Haryana, India, Te 91-999603।33।, Email drmittalpawan@gmail.com

Received: October 27, 2015 | Published: November 27, 2015

Keywords: diffuse axonal injury, beta amyloid precursor protein, immunostaining, white matter hemorrhages

Abbreviations: DAI, Diffuse Axonal Injury; H \& E, Hematoxylin-Eosin staining; B-APP, Beta Amyloid Precursor Protein

\section{Introduction}

Diffuse traumatic brain injury refers to widespread pattern of injury throughout the brain caused by traumatic disruption of nerve cells, with particular injury to the axons. ${ }^{1}$ There are four principal types of traumatic brain injury: diffuse vascular injury, diffuse axonal injury, hypoxic-ischemic encephalopathy and diffuse brain swelling. ${ }^{2}$ Clinically, DAI is characterized by generalized injury to the axons leading to immediate unconsciousness and coma longer than 6hours of duration. It is most commonly seen in road traffic fatalities and occasionally in assaults or fall from considerable height. ${ }^{3,4}$ Though the terms DAI and traumatic axonal injury have been used interchangeably, it has been proved that the diffuse injury to the axons can also occur in a variety of other non traumatic pathological conditions such as cerebral hypoxia, hypoglycemia etc. ${ }^{5-8}$ So the more specific term traumatic diffuse axonal injury or traumatic axonal injury has replaced diffuse axonal injury to specifically highlight axonal damage due to trauma. ${ }^{9}$ According to the severity of the pathological features three grades have been attributed to diffuse axonal injury. Grade I includes pathological features restricted to retraction balls or axonal swelling at characteristic sites in brain. Grade II comprising features of Grade I with hemorrhagic or necrotic lesions of corpus callosum along with involvement of interventricular septum and possibly intraventricular hemorrhage while Grade III involves lesions of dorsolateral area of rostral brainstem in addition. ${ }^{10}$

This pattern of distribution was however found quite unreliable in a subsequent study and variable frequencies of lesions were found in separate regions of brain. ${ }^{11}$ Presence of concomitant lesions in pons and corpus callosum has been found depictive of physical brain injury with pons being most frequent affected area. The medulla is comparatively spared. ${ }^{6}$ Pathologically the detection of diffuse axonal damage relies heavily on microscopic examination that explains duration dependent sequential axonal changes. H \& E staining and silver stains have been employed for the microscopic detection of diffuse axonal damage evident as axonal swellings or retraction balls. These histological techniques however require a significant post- injury survival period ranging from 12 to 36 hours. ${ }^{12}$ Furthermore, relatively poor visibility of injured neurons on $\mathrm{H} \& \mathrm{E}$ staining along with the underestimation of degree of axonal damage limits its use. ${ }^{10}$ B-APP immunostaining is the current gold standard detecting axonal changes at the earliest (less than 2hours) and has been found to be a sign of vital axonal damage. ${ }^{6}$ However the relative inability of these techniques to differentiate traumatic from non-traumatic causes of DAI on occasions may limit their practical utility.

\section{Diffuse vascular injury}

In severe DAI there is differential movement between different regions of brain leading to immediate tearing of axons along with small blood vessels at the site of movements. ${ }^{13}$ Damage to the blood vessels leads to multiple petechial haemorrhages within the brain predominantly in the white matter of frontal lobe (Figure 1). This vascular counterpart of diffuse axonal injury is termed as diffuse vascular injury. Most of the victims belong to motor vehicular injuries with very short survival time ranging from few minutes to some hours. ${ }^{14}$ DAI leads to unconsciousness wherein the victim remains in coma which may be the permanent vegetative state. ${ }^{15-18}$

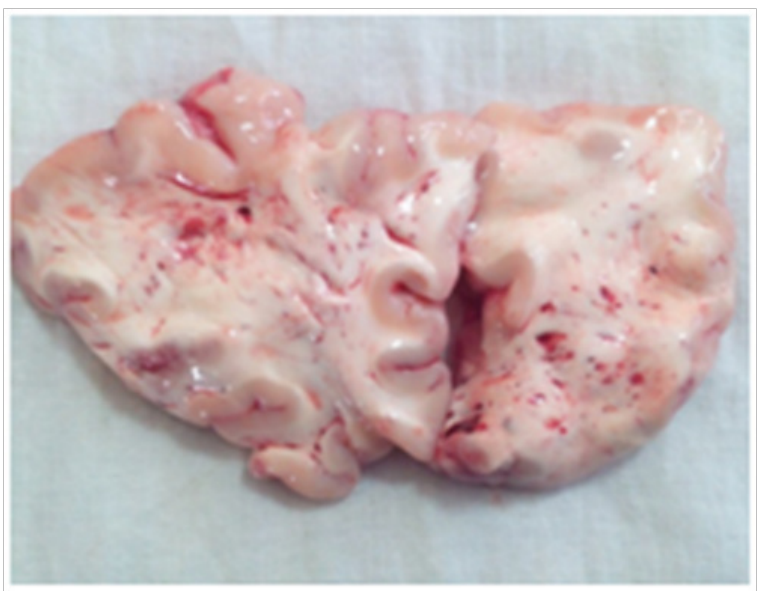

Figure I Petechial hemorrhages in frontal lobe white matter depictive of diffuse vascular injury (brain sectioned in fresh state). 


\section{Mechanism of brain trauma in DAI}

Experiments have revealed that the degree of acceleration and time span over which it occurs are the two important factors leading to diffuse axonal injury. Diffuse axonal injuries are characteristic of head acceleration occurring gradually over a lengthy time period. ${ }^{19}$ Furthermore head motion in the coronal plane was found to be more damaging leading to severe axonal damage rather than the sagittal motion that produced mild or at most moderate damage to axons..$^{20,21}$ This makes higher incidence of diffuse axonal injury in traffic fatalities in which the trauma is of absorbing nature over a period of time, unlike in falls and assaults which cause sudden decelerationacceleration of the head. Diffuse axonal injury and the accompanying tissue tear hemorrhages are considered to be due to high levels of local shear and tensile strains as well as strain rates as a result of rotational and translational head acceleration. ${ }^{22}$ These accelerationinduced tissue strains are associated with local brain displacements within the deep white matter which has been measured and calculated. At peak angular speeds of the head ranging from 17 to $22 \mathrm{rad} / \mathrm{s}$, peak accelerations of 10-150 gand peak angular accelerations of 1,000 to $8,000 \mathrm{rad} / \mathrm{s}^{2}$, it was noted that local motions of the brain with respect to the skull generally following loops or figure-of-eight patterns with peak displacements on the order of $+/-5 \mathrm{~mm}^{23,24}$

\section{Pathological features in DAI}

Macroscopic findings: Focal findings in diffuse traumatic brain injury may be in the form of non specific trauma to the skull, scalp, subarachnoid hemorrhage or cortical contusion. These are not considered necessary for the diagnosis of diffuse brain injury. ${ }^{1,2}$ The characteristic findings in DAI are hemorrhagic tears that are located in the deep long white matter tracts of brain involving corpus callosum, internal capsule and superior cerebellar peduncles. Gliding contusions of parasagittal frontal lobe white matter (Figure 2) and deep cerebral contusion of basal ganglia and region deep grey matter of brain, known as intermediary coup contusions (Figure 3), are characteristic of traumatic diffuse axonal injury. ${ }^{12,25}$ Widespread petechial haemorrahges in the centrum ovale region of brain predominantly in frontal lobe white matter region is depictive of diffuse vascular injury in severe closed head injury victims. ${ }^{14}$ The hemorrhages are not as prominent as those of diffuse axonal injury because enough time is not elapsed to allow them to develop as the victim rarely survives a few minutes. ${ }^{14,26}$

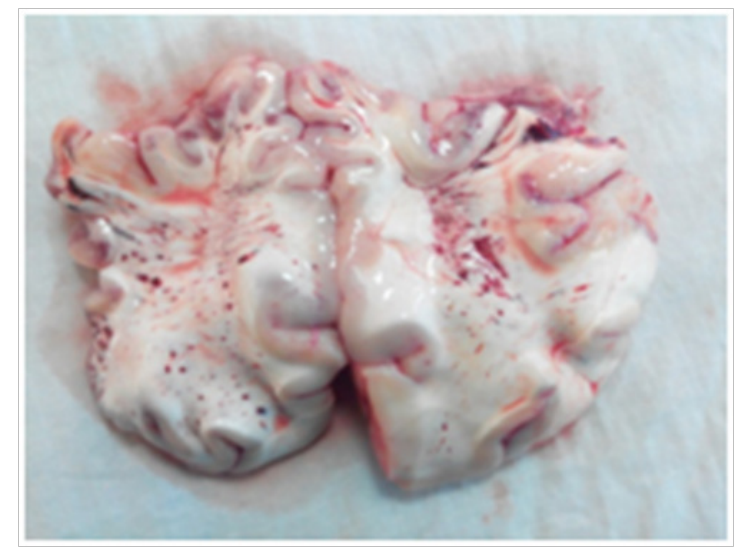

Figure 2 Petechial Hemorrhages, hemorrhagic tears and gliding contusions in parasagittal frontal lobe white matter along with intermediary contusion hematomas. Streaky contusion hemorrhages over frontal gyral crest also present (Brain Sectioned in Fresh State).

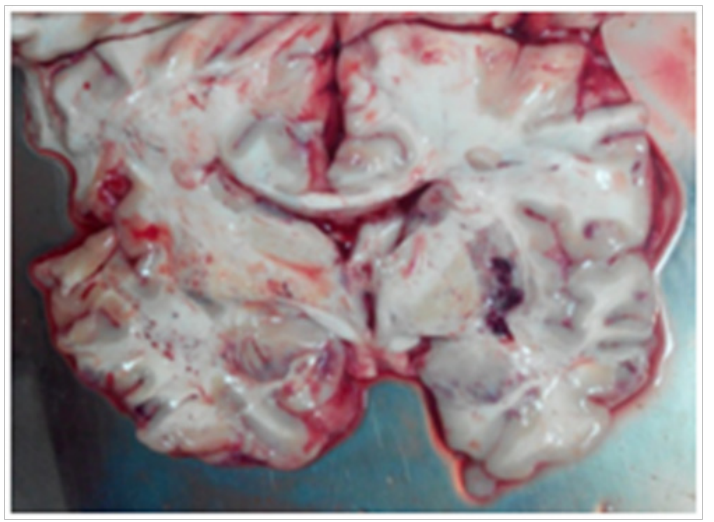

Figure 3 Contusion hematoma in right putamen region (basal ganglia) and white matter petechial hemorrhage in left temporal lobe (Brain Sectioned in Fresh State).

\section{Histology in DAI}

A number of histological techniques are available to appreciate sequential pathological changes in axons in diffuse axonal injury. These are primarily aimed at shortening the duration at which the axonal changes are seen and to put them in context to various traumatic and non traumatic conditions so as to differentiate the causative mechanism. Axonal swellings or retraction balls, representing transected axons, are the histological hallmark of axonal injury but are usually not visible before 24 to 36 hours by routine $\mathrm{H} \& \mathrm{E}$ staining or with a myelin stain like Luxol fast blue. ${ }^{2}$ Silver staining method can reliably demonstrate axonal swellings within 12 to 18 hours. ${ }^{27}$ The method has been found to be more sensitive and reliable as compare to $\mathrm{H} \& \mathrm{E}$ staining. However diffuse staining of axons by silver stains may occasionally make differentiation of injured and irregular axons difficult thereby limiting their practical utility. ${ }^{28}$ Injuries to the axons may be detected even earlier by immuno-histo-chemical methods such as by 6 hours using ubiquitin immunostaining. Persistent reactive axonal changes in the form of retraction balls were however more consistent 24 hours post-injury. A stronger immunoreactivity in the periphery was observed as compared to central region. ${ }^{29}$

B-APP immunostaining has been successful in demonstrating DAI in less than 2 hours. ${ }^{6}$ B-APP is normally present in the nerve cells but is not detected routinely due to its small quantity. However it accumulates as acute phase reactant in distended axons after injury and is visualized on routine and immunohistochemical examination. ${ }^{1}$ The main advantage of B-APP immunostaining over other is that $\mathrm{B}-\mathrm{APP}$ is accumulated within the axons even before other significant morphological axonal changes become apparent. ${ }^{27}$ From the visual pattern of injured axons there has been some success in differentiating microscopic changes characteristic of ischemia from trauma using B-APP immunostaining. Ischemic axons were found with wavy or zigzag pattern with abrupt differentiation of injured from healthy area while trauma causing scattered or group arrangement of axons in their long axis. ${ }^{9}$ However it is not uncommon for the traumatic and ischemic neuronal changes to coexist in the same case of trauma, making both the patterns to overlap significantly making reliable distinction between these two entities difficult at times. ${ }^{30}$ Electron microscopy has gained some success in defining axonal changes characteristic of trauma. ${ }^{31}$ Its use in routine forensic pathology is however limited. The etiology related non-specificity of the microscopic findings and the coexistence of traumatic and other neuropatho logical conditions in some victims may limit potential benefits of available histological and 
immunohistochemical techniques. A combination of these techniques when used in proper circumstances of the case may however prove beneficial in aiding the diagnosis.

\section{Dating DAl using microscopy}

With increase in the post injury survival time, there is an increase in extent and degree of axonal injury with axonal changes becoming more apparent on microscopy. Using silver staining there is significant change in the appearance of damaged neurons progressing from initial sinusoidal dilation at 2 hours to apparently large retraction balls at 72 hours. ${ }^{32}$ The maximum size of axonal swellings is achieved by approximately 85 hours after injury. ${ }^{33}$ Post trauma there is increase in the number of retraction balls during the first week due to further later degeneration of functioning but irreversibly injured axons. Two to three weeks later however there is decrease in the number of retraction balls with predominance of microglial cells followed by astrocytosis and demyelinization. ${ }^{34}$ After a period of 2 to 3months or longer a secondary bilateral wallerian degeneration becomes visible. ${ }^{35}$ Using neurofilament immunostaining it was made possible to determine duration of DAIs ranging from 30 minutes to greater than 24hours. ${ }^{36,37}$

The characteristics changes of axonal varicosities and retraction balls were found maximally in deep white matter and brain stem regions. ${ }^{37}$ Time since injury can be reliably estimated using B-APP immunostaining ranging from less than 3hours to greater than 6 months. There is slight positivity for B-APP during the earliest stage that progresses to strongly positive axonal bulbs then converting to fading positivity and finally a negative axonal reaction in cases of much longer duration. ${ }^{6,38,39}$ A relative fading or non staining of axons was found in midbrain and pons region..$^{39}$ Considering the selective regions of brain for histological evaluation of diffuse axonal injury and the changes of remote or organizing traumatic axonal change, results may be helpful in concluding about duration of injury where the victim dies weeks or months after sustaining head trauma.

\section{Concussion and diffuse axonal injury}

Concussion is trauma induced alteration in mental status with or without loss of consciousness..$^{40}$ Concussion is considered to be a manifestation of diffuse brain injury with little to no structural damage to brain. In cerebral concussion there is immediate loss of consciousness the duration of which may vary from minutes to hours persisting as confusion and is followed by posttraumatic retrograde amnesia. Mild concussion is characterized by confusion and disorientation but without loss of consciousness. Amnesia may or may not be present, typically developing 5 to 10 minutes after trauma with some residue remaining forever. The role of multifocal axonal injuries has been detected in cerebral concussion. Cases dying from other causes after incurring cerebral concussion have been reported on autopsy. Multifocal axonal injuries have been found on immunohistochemical staining with B-APP. ${ }^{4}$

\section{Differential diagnosis}

Occasionally the haemorrhages and petechiae of diffuse axonal and vascular injuries may be confused with other non traumatic pathological conditions like vascular congestion, petechiae of fat embolism, disseminated intravascular coagulation, air embolism, cerebral malaria etc. ${ }^{2}$ The etiology, location and distribution of these lesions along with the history and circumstances of the case often help differentiating traumatic from non traumatic condition.

\section{Conclusion}

The findings of diffuse traumatic brain injury may not be visible to the naked eye at autopsy. The evaluation requires extensive histological sampling of brain from various regions using variety of techniques. A victim suspicious of head trauma or otherwise may not show findings of any focal head trauma but may have died of diffuse axonal injury, becoming apparent within the brain matter in the form of hemorrhages and petechiae which requires proper brain sectioning. Furthermore microscopic findings in DAIs may not be etiology specific and thus require proper history regarding any preexisting neuropathological condition in trauma victims. It is also worth to mention that the customary practice of trivial external inspection of brain to look for any haemorrhage or pathology and subsequently making an opinion without sectioning is a classical mistake in forensic autopsy; a practice followed too often by some inexperienced pathologists. The damage to axons can only be appreciated if the brain has been sectioned properly; the same may be the only finding to the cause of death especially in a traffic victim.

\section{Acknowledgments}

None.

\section{Conflicts of interest}

The author declares that there are no conflicts of interest.

\section{References}

1. Dolinak D, Matshes EW, Lew EO. Forensic Pathology: Principles and Practice, 1st ed. Elsevier Academic Press, USA. 2005;442-446.

2. Shkrum MJ, Ramsay DA. Forensic Pathology of Trauma: Common Problems for the Pathologists. 1st ed. Humana Press, Totowa New Jersey. 2007;558-565.

3. Graham DI, Clark JC, Adam JH, et al. Diffuse axonal injury caused by assault. J Clin Pathol. 1992;45(9):840-841.

4. Adams JH, Doyle D, Graham DI, et al. Diffuse axonal injury in head injuries caused by a fall. Lancet. 1984;2(8417):1420-1422.

5. Geddes JF. What's new in the diagnosis of head injury? J Clin Path. 1997;50(4):271-274.

6. Oehmichen M, Meissner C, Schmidt V, et al. Axonal injury-a diagnostic tool in forensic neuropathology? A review. Forensic Sci Int. 1998;95(1):67-83.

7. Oehmichen $\mathrm{M}$, Meissner $\mathrm{C}, \mathrm{Schmidt} \mathrm{V}$, et al. Pontine axonal injury after brain trauma and nontraumatic hypoxic-ischemic brain damage. Int $J$ Legal Med. 1999;112(4):261-267.

8. Dolinak D, Smith C, Graham DI. Hypoglycaemia is a cause of axonal injury. Neuropathol Appl Neurobiol. 2000;26(5):448-453.

9. Geddes JF, Whitwell HL, Graham DI. Traumatic axonal injury: practical issues for diagnosis in medicolegal cases. Neuropathol Appl Neurobiol. 2000;26(2):105-116.

10. Adams JH, Doyle D, Ford I, et al. Diffuse axonal injury in head injury: definition, diagnosis and grading. Histopathology. 1989;15(1):49-59.

11. Blumbergs PC, Jones NR, North JB. Diffuse axonal injury in head trauma. J Neurol Neurosurg Psych. 1989;52(7):838-841.

12. DiMaio VJ, DiMaio D. Forensic pathology: Practical aspects of criminal and forensic investigations, 2nd ed. CRC Press, New York, USA. 2001;19 p. 
13. Povlishock JT. Traumatically induced axonal injury: pathogenesis and pathobiological implications. Brain Pathol. 1992;2(1):1-12.

14. Pittella JE, Gusmão SN. Diffuse vascular injury in fatal road traffic accident victims: its relationship to diffuse axonal injury. J Forensic Sci. 2003;48(3):626-630.

15. Adams JH, Graham DI, Murray LS, et al. Diffuse axonal injury due to nonmissile head injury in humans: an analysis of 45 cases. Ann Neurol. 1982;12(6):557-563.

16. Pilz P. Axonal injury in head injury. Acta Neurochir Suppl. 1983;32:119-123.

17. Strich SJ, Oxon DM. Shearing of nerve fibres as a cause of brain damage due to head injury: a pathological study of twenty cases. Lancet. 1961;278(7200):443-448.

18. Peerless SJ, Rewcastle NB. Shear injuries of the brain. Can Med Assoc J. 1967;96(10):577-582.

19. Wilberger JE Jr, Rothfus WE, Tabas J, et al. Acute tissue tear hemorrhages of the brain: computed tomography and clinicopathological correlations. Neurosurgery. 1990;27(2):208-213.

20. Adams JH, Graham DI, Gennarelli TA. Head injury in man and experimental animals: neuropathology. Acta Neurochir Suppl. 1983;32:15-30.

21. Gennarelli TA, Thibault LE, Adams JH, et al. Diffuse axonal injury and traumatic coma in the primate. Ann Neurol. 1982;12(6):564-574.

22. Povlishock JT, Christman CW. The pathobiology of traumatically induced axonal injury in animals and humans: a review of current thoughts. J Neurotrauma. 1995;12(4):555-564.

23. Hardy WN, Foster CD, Mason MJ, et al. Investigation of head injury mechanisms using neutral density technology and high-speed biplanar X-ray. Stapp Car Crash J. 2001;45:337-368.

24. Kleiven S, Hardy WN. Correlation of an FE model of the human head with local brain motion-consequences for injury prediction. Stapp Car Crash J. 2002;46:123-144.

25. Adams H, Mitchell DE, Graham DI, et al. Diffuse brain damage of immediate impact type. Its relationship to "primary brain-stem damage" in head injury. Brain. 1977;100(3):489-502.

26. Tomlinson BE. Brain-stem lesions after head injury. J Clin Pathol Suppl (R Coll Pathol). 1970;4:154-165.

27. McKenzie KJ, McLellan DR, Gentleman SM, et al. Graham DI. Is betaAPP a marker of axonal damage in short-surviving head injury? Acta Neuropathol. 1996;92(6):608-613.
28. Gleckman AM, Bell MD, Evans RJ, et al. Diffuse Axonal Injury in Infants with Nonaccidental Craniocerebral Trauma: Enhanced Detection by $\beta$-Amyloid Precursor Protein Immunohistochemical Staining. Arch Pathol Lab Med. 1999;123(2):146-151.

29. Schweitzer JB, Park MR, Einhaus SL, et al. Ubiquitin Marks the Reactive Swellings of Diffuse Axonal Injury. Acta Neuropathol. 1993;85(5): 503-507.

30. Smith C, Graham DI, Geddes JF, et al. The interpretation of beta-APP immunoreactivity: a response to C. Neiss et al. (2002) 104:79. Acta Neuropathol. 2003;106(1):97-98.

31. Erb DE, Povlishock JT. Axonal damage in severe traumatic brain injury: an experimental study in cat. Acta Neuropathol. 1988;76(4):347-358.

32. Vanezis P, Chan KK, Scholtz CL. White matter damage following acute head injury. Forensic Sci Int. 1987;35(1):1-10.

33. Wilkinson AE, Bridges LR, Sivaloganathan S. Correlation of survival time with size of axonal swellings in diffuse axonal injury. Acta Neuropathol. 1999;98(2):197-202.

34. Le Roux PD, Choudhri H, Andrews BT. Cerebral concussion and diffuse brain injury. In: Cooper PR, Golfinos TA, editors. Head Injury, 4th ed. McGraw-Hill, New York, USA. 2000.

35. Imajo T, Roessman U. Diffuse axonal injury. Am J Forensic Med Pathol. $1984 ; 5(3): 217-222$.

36. Grady MS, McLaughlin MR, Christman CW, et al. The use of antibodies targeted against the neurofilament subunits for the detection of diffuse axonal injury in humans. J Neuropathol Exp Neurol. 1993;52(2):143-145.

37. Li R, Fujitani N, Jia JT, et al. Immunohistochemical indicators of early brain injury: an experimental study using the fluid-percussion model in cats. Am J Forensic Med Pathol. 1998;19(2):129-136.

38. Gentleman SM, Roberts GW, Gennarelli TA, et al. Axonal Injury-a Universal Consequence of Fatal Closed-Head Injury. Acta Neuropathol. 1995;89(6):537-543.

39. Geddes JF, Vowles GH, Beer TW, et al. The diagnosis of diffuse axonal injury: implications for forensic practice. Neuropathol Appl Neurobiol. 1997;23(4):339-347.

40. Kelley JP, Nichols JS, Filley CM, et al. Concussion in sports: Guidelines for the prevention of catastrophic outcome. JAMA. 1991;266(20):2867-2869.

41. Blumberg PC, Scott G, Manavis J, et al. Staining of amyloid precursor protein to study axonal damage in mild head injury. Lancet. 1994;344(8929):1055-1056. 